Die Folgen von Informations- und Kommunikations-Techniken für die Virtualisierung der Wirtschaft

\section{Bits statt Atome?}

\section{Wirtschaftliche Prozesse werden zunehmend durch Informations- und Kommu- nikations(luK)-Aktivitäten geprägt. Was bedeutet die Leisłungsexplosion im Bereich von Informations- und Kommunikationstechnik für Markt- und Unter- nehmensstrukturen? Und welche ökologischen und sozialen Trends sind damit verbunden?}

$\mathrm{G}$

Von Arnold Picot ewichtige Argumente sprechen dafïr, daß die rasante Entwicklung der IuK-Technologien zu einer Belebung des Wettbewerbs führt; u.a. genannt werden mehr Markttransparenz, erleichterter weltweiter Marktauftritt über das Internet, geringere Wechselkosten für den Nachfrager sowie sinkende Transaktionskosten in vielen Bereichen.

Aber die Gegenkräfte sind auch nicht zu übersehen, u.a.:

- neue „economies of scale" im Bereich der Informations- und Medienproduktion und -distribution sowie der Systementwicklung und -nutzung,

- Netz- und Lock-in-Effekte aufgrund von im Wettbewerb durchgesetzten de-facto-Standards,

- neuartige vertikale Verbundsysteme mit erschwertem Marktzutritt für Dritte.

Vermutlich wird beides zugleich auftreten. Wir werden wohl vermehrt solche Felder sehen, auf denen sich der Wettbewerb innerhalb und zwischen Branchen und Regionen verschärft und wo neue Marktteilnehmer die Dynamik des Wettbewerbs sowohl im Bereich des Technologieangebots als auch vor allem bei der Technologieanwendung steigern. Das wird für alle anstrengender, enthält aber interessante Chancen für neues, bewegliches Unternehmertum. Daneben werden einige Bereiche weiter rapide an Bedeutung gewinnen, in denen die Konzentration und Hierarchisierung der Märkte voranschreitet durch Ausschöpfung hoher Skalenerträge und Netzeffekte, durch enge Kooperationsnetze und Ausschöpfung anderer Verbundvorteile. Ein solcher Befund zieht - wie jede Veränderung von Wettbewerbsstrukturen - beträchtliche regionale, branchenbezogene und personelle Verteilungswirkungen nach sich.

Die zunehmende Durchdringung der Wirtschaft mit IuK-Techniken hat aber noch weitere mögliche Implikationen, die u.a. auch ökologisch und sozial von Interesse sind. Das Ende der Entfer- nung als Einflußgröße der Kommunikationskosten (sinkende Telekommunikationskosten, "flat rates"), die zunehmende Standortverteilung und Standortunabhängigkeit wirtschaftlicher Aktivitäten und die Verlagerung vieler Funktionen auf eine rein elektronisch-informationelle Ebene (Internet, Multimedia) führen zu ganz anderen, viel weniger physisch und räumlich gebundenen Leistungsstrukturen. Daher liegt die These nahe, daß sich die Wirtschaft immer mehr virtualisiert und infolgedessen auch stärker entmaterialisiert und entpersönlicht.

In der Tat gibt es auch für diese Überlegung verschiedene überzeugende Begründungen und Beobachtungen:

\section{- Telearbeit, mobile Arbeit,}

- standortverteilte, telekommunikativ vernetzte Projektteams,

- dynamische Unternehmensnetzwerke, die sich kundenauftrags- und problemabhängig zu Wertschöpfungsgemeinschaften zusammenfinden, um sich alsbald wieder aufzulösen,

- Einkauf aus elektronischen Katalogen und virtuellen Shoppingmalls im Internet,

- Transport von Bits statt Atomen, wie Negroponte es nennt (1),

- Fernwartung, Teleservice und

- Bedienung durch Automaten.

Sehr viele Produkte sind unter dem Einfluß der Mikroelektronik und Digitalisierung informationsintensiver und materialärmer, d.h. auch leichter geworden, und viele Produkte lassen sich über Draht transportieren, ehe sie physisch vor Ort verfiigbar gemacht werden (z.B. Druckerzeugnisse, CDs). Insofern könnte die Virtualisierung zu Energie- und Materialersparnis führen. Aber ist das eine Einbahnstraße? Erzeugt die Entwicklung nicht auch neuen Bedarf und neue Chancen für physischen Transport, für Anfaßbares und Persönliches? Werden wir wirklich weniger reisen, uns seltener persönlich begegnen, weniger transportieren, weniger Energie und
Materie benötigen? Es gibt zumindest vier Kräfte, die einem generellen Trend in diese Richtung entgegenwirken, ja diesen vielleicht per Saldo in manchen Fällen umkehren:

\section{- Grenzen der Entmaterialisierung}

1. Langzeitstudien zeigen, daß Information und Kommunikation auch Organisatoren des physischen Transports sind, also Transporte per Saldo nicht substituieren, sondern generieren. Information und Kommunikation eilen den Bewegungen von Menschen und Gïtern voraus, bereiten sie vor, regen sie an. Ohne Vorausinformation gehen nur wenige Abenteurer auf Reisen. Mit breit verfiugbarer und qualifizierter Information bewegen sich halbe Kontinente Sommer wie Winter für einige Wochen in weit entfernte Urlaubsparadiese. Natïrlich kommt die Transporttechnik hinzu, aber ohne die Information, die Abstimmung usw. würden diese massenhaften Transporte nicht stattfinden. Ähnliches gilt für die Anbahnung von Geschäften und für Warenbewegungen. Somit sei die These erlaubt, daß die radikalen Kostensenkungen im Bereich von Information und Kommunikation, die jetzt bevorstehen, per Saldo eher mehr als weniger Transportaktivitäten von Menschen und Waren auslösen werden, insbesondere, wenn die Transportkosten konstant bleiben oder gar sinken.

2. Die Entlastungs- und Erleichterungseffekte, die sich für wirtschaftliche Akteure mit der IuK-Technik einstellen, haben, wie u.a. eine Studie von Pribilla/ Reichwald/ Goecke zeigt (2), entgegen den üblichen Annahmen nicht unbedingt den Effekt, die Reisen von Managern zu reduzieren. Vielmehr reisen gerade Manager, die sich der Technik besonders intensiv bedienen, auch besonders viel. Dies wurde von den Autoren als das Telekommunikationsparadox bezeichnet. Eine der mehreren möglichen Erklärungen möchte ich anbieten: Face-to-face-Kontakte sind für bestimmte Situationen unerläßlich (z.B. Vertrauen aufbauen, Chancen und Risiken bewerten, Commitment herstellen, persönliche Angelegenheiten erörtern). An jeden Manager wird von Kunden, externen Partnern und Unternehmensmitgliedern eine Übernachfrage nach solchen Kontakten gerichtet. Sofern es nun möglich ist, den Informationsauștausch mit der vertrauten Homebase durch leistungsfähige IuK-Technik zu erledigen, können mehr als zuvor derartige Anforderungen vor Ort, also außerhalb des eigenen Bürostandorts wahrgenommen werden. Das ist umso notwendiger, je standortverteilter, ja globaler Kunden, Lieferanten, 
Unternehmenseinheiten und sonstige Partner angesiedelt sind, was ja wiederum eine Folge der IuK-Durchdringung ist. Dieser Zusammenhang gilt natïrlich nicht nur für die erwähnten Manager, sondern für nahezu alle wirtschaftlichen Akteure, die sich in analogen Situationen befinden, also etwa auch für Fachkräfte, Repräsentanten von Lieferanten und Kunden usw.

3. Virtuellen Organisationsformen wird häufig ein sog. Vertrauensdilemma nachgesagt. Demnach sind standortverteilte Kooperationen, in denen eben nicht alles durch umfassende, sondern lediglich durch unvollständige Verträge geregelt werden kann, einerseits in besonderem Maße auf gegenseitiges Vertrauen angewiesen, andererseits aber bietet die Distanz oder die Begegnung im Netz kaum Möglichkeiten des Vertrauensaufbaus, weil dieser bekanntermaßen der persönlichen Begegnung bedarf. Gerade deshalb kommt es vermehrt zu Reiseaktivitäten. Eine Gründerin einer Internet-Büroservice-Agentur wies darauf hin, wie häufig sie auf Reisen zu Kunden sei, um den laufenden Geschäftsbetrieb über das Netz in Gang zu bringen. Erst wenn die Reputation sehr groß und die positive Erfahrung mit weitgehend standardisierten Leistungsqualitäten konsolidiert ist, kann ein guter Namé in einem virtuellen Geschäft die persönliche Begegnung als Vertrauensgrundlage einer Geschäftsbeziehung ersetzen. Ohne Zweifel werden Reputationsmechanismen in der onlineWelt noch viel wichtiger als heute ohnehin schon. 4. Zudem ist zu beobachten, daß gerade Unternehmen, die $\mathrm{zu}$ ihren Kunden ausschließlich eine gleichsam unsichtbare virtuelle Beziehung haben (z.B. Kreditkartenunternehmen, Telekommunikationsanbieter) zur Profilierung bewußt traditionelle materielle Komponenten wie persönliche Anschreiben, Kundenzeitschriften 0. ä. einsetzen. Internetunternehmen werben in traditionellen Printmedien und unterhalten Hotlines bzw. Callcenter für persönliche Anfragen und Beratungen. Offensichtlich gibt es also eine nützliche Komplementarität zwischen dem Cyberspace und der alten Medienwelt. Die Deutsche Post AG erlebt seit einigen Jahren einerseits einen Rückgang bei der traditionellen Briefpost, andererseits aber trotz Fax, Email und Internet hohe Wachtumsraten bei der Infopost und beim Zeitungsdienst. Von dem Boom bei Telefon und Mobilfunk ganz zu schweigen. Und wer hat trotz aller Elektronik das oft vorhergesagte papierlose Büro schon einmal gesehen? Zusammenfassend ist festzuhalten: Es besteht kein Zweifel, daß virtualisierte Formen der Organisati- on im Sinne standortverteilter und/ oder netzbasierter Leistungserstellung und Leistungspräsentation, daß die Transporte von Bits statt von Atomen rapide zunehmen werden. Der Stoff- und Energieeinsatz pro Leistungseinheit verringert sich, und das ist natülich positiv zu werten. Aber es ist $\mathrm{zu}$ vermuten, daß sich die Zahl der Leistungseinheiten aufgrund der Rationalisierungs- und Vereinfachungseffekte erhöht. Es gibt zudem einen steigenden Bedarf nach persönlicher Nähe und auch nach kurzer Entfernung zu möglichst vielen potentiellen Gesprächspartnern, wie das Wachstum bestimmter wirtschaftlicher Ballungsräume belegt. Es beweist sich eine alte Erkenntnis: Neue Medien haben noch nie in der Geschichte der Menschheit die alten vollständig abgelöst, sondern diese allenfalls peripher substituiert. Sie gehen mit den alten Medien neuartige Verbindungen ein und zwingen sie zur Neupositionierung. Genau das beobachten wir gegenwärtig in sehr vielen Bereichen.

\section{- Ökologische Auswirkungen}

Entscheidende ökologische Effekte der IuKTechnologien liegen also nicht primär auf der Ebene der Substitution physischer und persönlicher Verkehre, die sogar eher noch ausgedehnt werden, freilich bei zugleich gesunkenen durchschnittlichen Stiickkosten sowie erheblichen, breiten Wohlfahrtseffekten. Allerdings gibt es ökologisch relevante indirekte, positive Wirkungen der IuK-Technik, die nicht zu unterschätzen sind, nämlich insbesondere:

- eine raschere Diffusion auch ökologisch relevanten Wissens und entsprechender Technologien über die weltweite Vernetzung,

- zum Teil drastisch verbesserte Meß- und Kontrollmöglichkeiten von Umwelteinwirkungen und Standards aufgrund der enormen Verbesserungen der von der IuK-Technik getriebenen PreisLeistungs-Relationen bei der Erfassung und Analyse von Emissionen, Immissionen, Rückständen und Veränderungen nahezu aller Art.

- höhere Markttransparenz und verbesserte Informationsunterstiitzung bei der Aushandlung und Durchsetzung ökologischer Aspekte im Kauf- bzw. Verkaufsprozeß auch im globalen Kontext sowie

- eine erleichterte Abstimmung von Interessengruppen aller Art, insbesondere auch von Verbrauchern und Umweltverbänden.

Daß wir uns in einer Transformation der Wirtschaft unter dem Einfluß der IuK-Technik befinden, ist kaum bestreitbar. Diese Transformation beginnt allerdings gerade erst und wird an
Momentum zunehmen in Richtung auf mehr Wettbewerb und mehr Konzentration zugleich, mehr Chancen für neues Unternehmertum, aber auch erhebliche Risiken für tradierte Unternehmen, Branchen und Regionen, mehr Virtualisierung und Entmaterialisierung vieler Geschäftsbeziehungen und Leistungsprozesse, aber zugleich auch neue Möglichkeiten und Nachfrage für persönliche Kontakte, physische Transporte und traditionelle Medien sowie nicht zuletzt auch in Richtung von wesentlich verbesserter umweltbezogener Koordination aller Akteure.

Wie die beiden Enquete-Kommissionen ,Schutz des Menschen und der Umwelt“ und „Zukunft der Medien in Wirtschaft und Gesellschaft" des Deutschen Bundestages der letzten Legislaturperiode übereinstimmend festgestellt haben (3), umfaßt das Konzept der Nachhaltigen Entwicklung ein ,magisches“ Dreieck zwischen Ökonomie, Ökologie und sozio-politischer Ebene. Der überlegte, innovative und intensive Einsatz der IuK-Technik kann per Saldo alle drei Dimensionen der Nachhaltigkeit fördern, im Einzelfall allerdings mit z.T. gegenläufigen Effekten.

\section{Anmerkungen}

(1) Negroponte, N.: Being digital, New York 1995.

(2) Pribilla, P., R. Reichwald, R. Goecke: Telekommunikation im Management: Strategien für den globalen Wettbewerb, Stuttgart 1996.

(3) Vgl. Enquete-Kommission "Schutz des Menschen und der Umwelt" des 13. Deutschen Bundestages: Konzept Nachhaltigkeit. Vom Leitbild zur Umsetzung. Deutscher Bundestag, Referat für Öffentlichkeitsarbeit; Zur Sache $4 / 98$ und

Enquete-Kommission „Zukunft der Medien in Wirtschaft und Gesellschaft - Deutschlands Weg in die Informationsgesellschaft" des 13. Deutschen Bundestages. Deutscher Bundestag, Hrsg.: Verbraucherschutz in der Informationsgesellschaft, 1998.

\section{Der Autor}

Prof. Dr. Arnold Picot ist Vorstand des Instituts für Organisation an der Ludwig-Maximilians-Universität München. Er war Mitglied in der Enquete-Kommission "Zukunft der Medien in Wirtschaft und Gesellschaft" des 13. Deutschen Bundestages.

Kontakt: Institut für Organisation, Ludwigstr. 28, 80539 München. Tel. 089/21 80-22 52, Fax -3685 E-mail: Picot@Bwl.Uni-München.de 
(c) 20I0 Authors; licensee IÖW and oekom verlag. This is an article distributed under the terms of the Creative Commons Attribution Non-Commercial No Derivates License (http://creativecommons.org/licenses/by-nc-nd/3.o/), which permits unrestricted use, distribution, and reproduction in any medium, provided the original work is properly cited. 